\title{
Activity-Dependent Dynamic Microtubule Invasion of Dendritic Spines
}

\author{
Xindao Hu, ${ }^{1}$ Chris Viesselmann, ${ }^{2}$ Sookin Nam, ${ }^{2}$ Elliott Merriam, ${ }^{1}$ and Erik W. Dent ${ }^{1,2}$ \\ ${ }^{1}$ Neuroscience Training Program and ${ }^{2}$ Department of Anatomy, University of Wisconsin-Madison, Madison, Wisconsin 53706
}

Dendritic spines are the primary sites of contact with presynaptic axons on excitatory hippocampal and cortical neurons. During development and plasticity spines undergo marked changes in structure that directly affect the functional communication between neurons. Elucidating the cytoskeletal events that induce these structural changes is fundamental to understanding synaptic biology. Actin plays a central role in the spine cytoskeleton, however the role of microtubules in spine function has been studied little. Although microtubules have a prominent role in transporting material throughout the dendrite that is destined for spines, they are not thought to directly influence spine structure or function. Using total internal reflectance fluorescent microscopy we discovered that microtubules rapidly invade dendritic protrusions of mature CNS neurons (up to $63 \mathrm{~d}$ in vitro), occasionally being associated with marked changes in spine morphology in the form of transient spine head protrusions (tSHPs). Two microtubules can occupy a spine simultaneously and microtubule targeting can occur from both the proximal and distal dendrite. A small percentage of spines are targeted at a time and all targeting events are transient, averaging only a few minutes. Nevertheless, over time many spines on a dendrite are targeted by microtubules. Importantly, we show that increasing neuronal activity enhances both the number of spines invaded by microtubules and the duration of these invasions. This study provides new insight into the dynamics of the neuronal cytoskeleton in mature CNS neurons and suggests that microtubules play an important, direct role in spine morphology and function.

Key words: cytoskeleton; activity; hippocampus; cortex; synapse; plasticity

\section{Introduction}

During development spines undergo marked changes in structure ranging from motile filopodial protrusions to stable mushroom-shaped spines. The morphology of spines can directly affect functional communication between neurons (Hayashi and Majewska, 2005; Alvarez and Sabatini, 2007; Bourne and Harris, 2007; Harms and Dunaevsky, 2007). Enlarged spine heads correlate with an increased size of the postsynaptic density, whereas simplification of spine morphology and loss of entire dendritic spines can occur in developmental and adult neurological disorders, including mental retardation, autism and epilepsy (Calabrese et al., 2006; Tada and Sheng, 2006). Actin filaments play prominent roles in the formation, maintenance and plasticity of dendritic spine structure (Ethell and Pasquale, 2005). However, the role of microtubules (MTs) in spine architecture has been studied little. Generally, spines are thought to be devoid of MTs because electron micrographs have shown MTs in spines only in extremely rare circumstances (Gray et al., 1982; Chicurel and Harris, 1992; Fiala et al., 2003). Furthermore, live-cell imaging with fluorescently labeled microtubule associated proteins

Received June 3, 2008; revised 0ct. 14, 2008; accepted 0ct. 16, 2008.

This work was supported by start-up funds from the University of Wisconsin (UW) Department of Anatomy, the UW Graduate School, the UW School of Medicine and Public Health, and the UW Center for Neuroscience. We thank Katherine Kalil for the use of the Amaxa electroporator, Yu Huang and Justin Williams for the production of the perfusion insert, and Camin Dean for advice during early stages of the work.

Correspondence should be addressed to Erik W. Dent, Department of Anatomy, University of Wisconsin-Madison, 1300 University Avenue, 332 SMI, Madison, WI 53706. E-mail: ewdent@wisc.edu.

DOI:10.1523/JNEUROSCI.3074-08.2008

Copyright $\odot 2008$ Society for Neuroscience $\quad 0270-6474 / 08 / 2813094-12 \$ 15.00 / 0$ has failed to detect the presence of MTs in spines (Kaech et al., 2001).

Prominent in dendrite shafts, MTs are assumed to function exclusively as stable railways for long distance intracellular transport. However, MTs exhibit bouts of rapid polymerization and depolymerization, termed dynamic instability (Mitchison and Kirschner, 1984). MTs have been shown to rapidly polymerize into and depolymerize from all regions of developing neurons (Stepanova et al., 2003), including the tips of growth cone filopodia (Dent and Kalil, 2001; Schaefer et al., 2002). We hypothesized that MTs remain dynamic in mature neurons and are capable of exploring intracellular space through dynamic polymerization and depolymerization. To test this idea we imaged MTs in dendrites of mature hippocampal and cortical neurons, fluorescently labeled with either EGFP- $\alpha$-tubulin or EB3EGFP. EGFP- $\alpha$-tubulin incorporates throughout MTs allowing us to image all microtubules within a living neuron. However, EB3-EGFP, a MT plus end tracking protein (+TIP), labels the fast growing plus ends of polymerizing MTs, but not paused or depolymerizing MTs (Stepanova et al., 2003). We can document MT dynamics (polymerization, depolymerization, pausing) in dendritic protrusions with EGFP- $\alpha$-tubulin-labeled MTs, whereas imaging EB3-EGFP-labeled plus ends of MTs allow us to follow MT polymerization in both the shaft and protrusions.

We used total internal reflection fluorescence microscopy (TIRFM) to track MT dynamics in spines that were positioned near or on the coverslip. TIRFM is a laser-based microscopy technique that only illuminates $\sim 100-400 \mathrm{~nm}$ above the glass sub- 
strate (Schneckenburger, 2005). We obtained high signal-tonoise images of MTs entering micron-sized structures (dendritic protrusions) and imaged two fluorophores at rapid intervals (10 s) for long periods of time $(1 \mathrm{~h})$. Surprisingly, we discovered that MTs are capable of extending rapidly into and out of dendritic protrusions (filopodia and spines) and remain dynamic throughout neuronal development (up to DIV 63). Importantly, we also discovered that MT invasion of dendritic protrusions was dependent on neuronal activity. These results indicate that MTs may have a hitherto unappreciated role as a central player in spine function.

\section{Materials and Methods}

Plasmids and reagents. GFP- $\alpha$-tubulin (human) in a pCAX vector and mCherry- $\beta$-actin (human) in a C1 vector (Clontech) have been described previously (Dent et al., 2007). DsRed2 (Clontech) was cloned into a pCAX vector (Osumi and Inoue, 2001) using standard techniques. EB3-EGFP was a generous gift from Niels Galjart (Erasmus Medical Center, Rotterdam, Netherlands). Newly polymerized, dynamic microtubules were labeled with rat anti-tyrosinated tubulin at 1:1000 (YL1/2 clone, Millipore). Presynaptic terminals were labeled with antisynaptophysin at 1:5000 (Millipore). All Alexa-conjugated secondary antibodies and Alexa-conjugated phalloidin, to label actin filaments, were from Invitrogen.

Hippocampal and cortical neuron culture and transfection. All mouse procedures were approved by the University of Wisconsin Committee on Animal Care and were in accordance with NIH guidelines. Cortical (E14.5-16.5) and hippocampal (E15.5-17.5) neuron cultures were prepared from Swiss Webster mice (Taconic) essentially as described (Lebrand et al., 2004; Dent et al., 2007). Briefly, cortices and hippocampi were dissected, trypsinized and dissociated. Dissociated cortical or hippocampal neurons were resuspended in Nucleofector solution (Mouse Neuron Kit, Amaxa Biosystems) and transfected with an Amaxa Nucleofector according to the manufacturer's directions. Transfected neurons were plated at densities of $2-4 \times 10^{4}$ neurons $/ \mathrm{cm}^{2}$ on $1.0 \mathrm{mg} / \mathrm{ml}$ poly-D-lysine (Sigma)-coated glass coverslips adhered to the bottom of $35 \mathrm{~mm}$ plastic culture dishes that had a $15 \mathrm{~mm}$ hole drilled through the bottom of the chamber. Neurons were plated in plating medium (PM) which consisted of Neurobasal medium with 5\% FBS (Hyclone), B27 supplement, $2 \mathrm{~mm}$ glutamine, $37.5 \mathrm{~mm} \mathrm{NaCl}$ and $0.3 \%$ glucose. After $1 \mathrm{~h}$, this medium was replaced with serum-free medium (SFM), which was PM without FBS.

Live-cell TIRF imaging. The TIRFM microscope consisted of a Nikon TE2000E base with TIRF illuminator (Nikon), a Nikon $100 \times / 1.49 \mathrm{NA}$ Plan Apo TIRF objective, a Nikon perfect focus system for continuous automatic focusing of the sample during time-lapse imaging, a Nikon $\mathrm{z}$-motor, a motorized $\mathrm{x}$-y stage (Prior Scientific), a Lumen Pro200 fluorescent illumination system consisting of a $200 \mathrm{~W}$ metal halide lamp and six-position excitation filter wheel and a fiber optic illuminator (Prior Scientific), a 10-position emission filter wheel and a Coolsnap HQ cooled interline CCD camera (Photometrics). For TIRF illumination two lasers were used: a $40 \mathrm{~mW}$ Argon laser for GFP illumination and a $10 \mathrm{~mW}$ solid state $561 \mathrm{~nm}$ laser for DsRed2 and mCherry illumination (both MellesGriot). Laser power was attenuated to $6.25 \%$ with neutral density filters. The microscope was equipped with a dual wavelength (EGFP/mCherry) dichroic mirror (z488/561rdc, Chroma) for both TIRF and wide-field illumination. Wide-field fluorescent illumination was accomplished with the aid of a Lumen Pro 200 (Prior Scientific), containing an excitation filter wheel with GFP and mCherry excitation filters, a metal halide illuminator and a liquid light guide. This system allowed us to collect two-color TIRF, two-color widefield and a DIC image in quick succession. Usually, we only collected wide-field and DIC images at the beginning and end of a time-lapse. All images were collected, measured and compiled in MetaMorph imaging software (Molecular Devices). Figures were compiled in Photoshop (Adobe). During time-lapse microscopy neurons were kept at $37^{\circ} \mathrm{C}$ with in an incubation chamber (Solent Inc.) custom fitted to the microscope. For fluorescent live-cell imaging the chamber was closed with a glass ring, coverslip and silicone grease.

Induction of activity in hippocampal cultures. To induce global activa- tion of hippocampal cultures we briefly added high concentrations of $\mathrm{KCl}$ to the cultures essentially as described (Li et al., 2004). We modified this approach for both live-cell and fixed-cell imaging. Briefly, for livecell imaging we slowly exchanged the SFM media with ECS $(140 \mathrm{~mm}$ $\mathrm{NaCl}, 5 \mathrm{~mm} \mathrm{KCl}, 2 \mathrm{~mm} \mathrm{CaCl}$, 2 mм $\mathrm{MgCl}_{2}, 5 \mathrm{~mm}$ HEPES, $20 \mathrm{~mm}$ Glucose, $315 \mathrm{mOsm}$ ) of the same osmolarity and temperature as SFM in cultures that were previously transfected with EGFP- $\alpha$-tubulin/DsRed2. A custom silicone insert was placed in the $35 \mathrm{~mm}$ dish to allow for rapid addition/removal of media and an inlet and outlet (Bioscience Tools) were positioned in the dish. After 30 min of imaging in ECS, ECS with 75 $\mathrm{mm} \mathrm{KCl}$ (osmolarity balanced by removing $\mathrm{NaCl}$ ) was manually perfused into the chamber and left for $3 \mathrm{~min}$. Excess media was removed with a small vacuum pump. Fresh ECS was exchanged for the high KCl-ECS and left for $10 \mathrm{~min}$. This was repeated three times in total. Neurons were then imaged for an additional $30 \mathrm{~min}$. For fixed cell imaging we slowly exchanged SFM for ECS and let the cells recover for $10 \mathrm{~min}$. We then

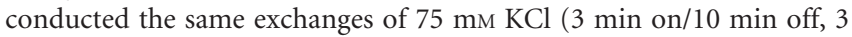
times) and then fixed the cultures $20 \mathrm{~min}$ after the final $\mathrm{KCl}$ removal for quantitation of MT invasion in dendritic protrusions. Controls for both experiments included all of the same exchanges with ECS instead of ECS with $75 \mathrm{~mm} \mathrm{KCl}$. In some experiments $1 \mu \mathrm{M}$ tetrodotoxin (TTX) was included in all solutions (with or without $\mathrm{KCl}$ ).

Quantitative analysis of protrusions and MT kinetics. All MT measurements were made with the aid of MetaMorph software (Molecular Devices). Morphological criteria for determining protrusion type were essentially as described (Chapleau et al., 2008). Briefly, spines were defined as protrusions $<3 \mu \mathrm{m}$ in length, whereas filopodia were $3-10 \mu \mathrm{m}$ in length. For all protrusions the head width at widest point $(\mathrm{H})$, neck width at the junction of the protrusion and dendrite $(\mathrm{N})$ and protrusion length from dendrite/protrusion junction to distal extent of protrusion (L) were measured with MetaMorph software. Stubby spines were defined as having parameters $(\mathrm{H} \sim \mathrm{N} \sim \mathrm{L})$, thin spines were defined as $(\mathrm{H} \sim \mathrm{N}, \mathrm{H}<\mathrm{L}$, $\mathrm{N}<\mathrm{L})$ and mushroom-shaped spines were defined as $(\mathrm{H}>\mathrm{N}, \mathrm{H} \sim \mathrm{L})$. Filopodia were longer than spines and did not possess an enlargement (head) at their distal ends. Live-cell images were collected at a resolution of $0.126 \mu \mathrm{m} /$ pixel. All images used for quantifications $(n=69$ spines, $n=23$ filopodia) were collected at $10 \mathrm{~s}$ intervals for a period of $1 \mathrm{~h} \mathrm{(361}$ images). This collection scheme resulted in 33,212 quantifiable images. MT invasion ratios (Fig. 1 ) were calculated by taking the ratio of the length of MT invasion into the protrusion divided by the protrusion length (DsRed2 channel). A protrusion ratio of 1.0 indicates the MT extended to the tip of the protrusion. Numbers $>1.0$ indicate the formation of a transient spine head protrusion (tSHP) in which the length to the distal tip of the spine head proper is used as the denominator.

Immunocytochemistry. Neurons were fixed in $4 \%$ paraformaldehyde/ KREBs/sucrose or $4 \%$ paraformaldehyde/PHEM medium at $37^{\circ} \mathrm{C}$ (Dent et al., 2007). Cultures were rinsed in PBS and blocked with $10 \%$ BSA/ PBS, permeabilized in $0.2 \%$ Triton X-100/PBS and labeled with primary (see above) and secondary antibodies at 1:500 (Invitrogen). Phalloidin coupled to Alexa 350, 488 or 568 (Invitrogen) was used to label actin filaments (1:25 to 1:100). Images of fixed and labeled neurons were collected in both widefield and TIRFM at a resolution of $0.063 \mu \mathrm{m} /$ pixel.

Statistics and graphing. All statistical tests were performed with GraphPad Prism 4.0c. For each data set parametric or nonparametric statistical tests and post hoc tests were performed depending on the variance of the data sets. Graphs were compiled in Microsoft Excel or Prism.

\section{Results}

To determine whether and how MTs are capable of entering dendritic protrusions we imaged MT dynamics with time-lapse TIRFM. Because TIRFM only excites fluorophores within a few hundred nanometers of the cell/coverslip interface we were able to acquire high signal-to-noise images of MT dynamics in spines within dense neuronal cultures (Fig. $1 e-g$ ). Furthermore, TIRF imaging allowed us to image sections of dendrites labeled with EGFP- $\alpha$-tubulin and DsRed2 (as a volume marker) at rapid intervals ( $10-30 \mathrm{~s}$ intervals) for extended periods of time $(8-20 \mathrm{~h})$, without any obvious deleterious effects to the neurons (supple- 


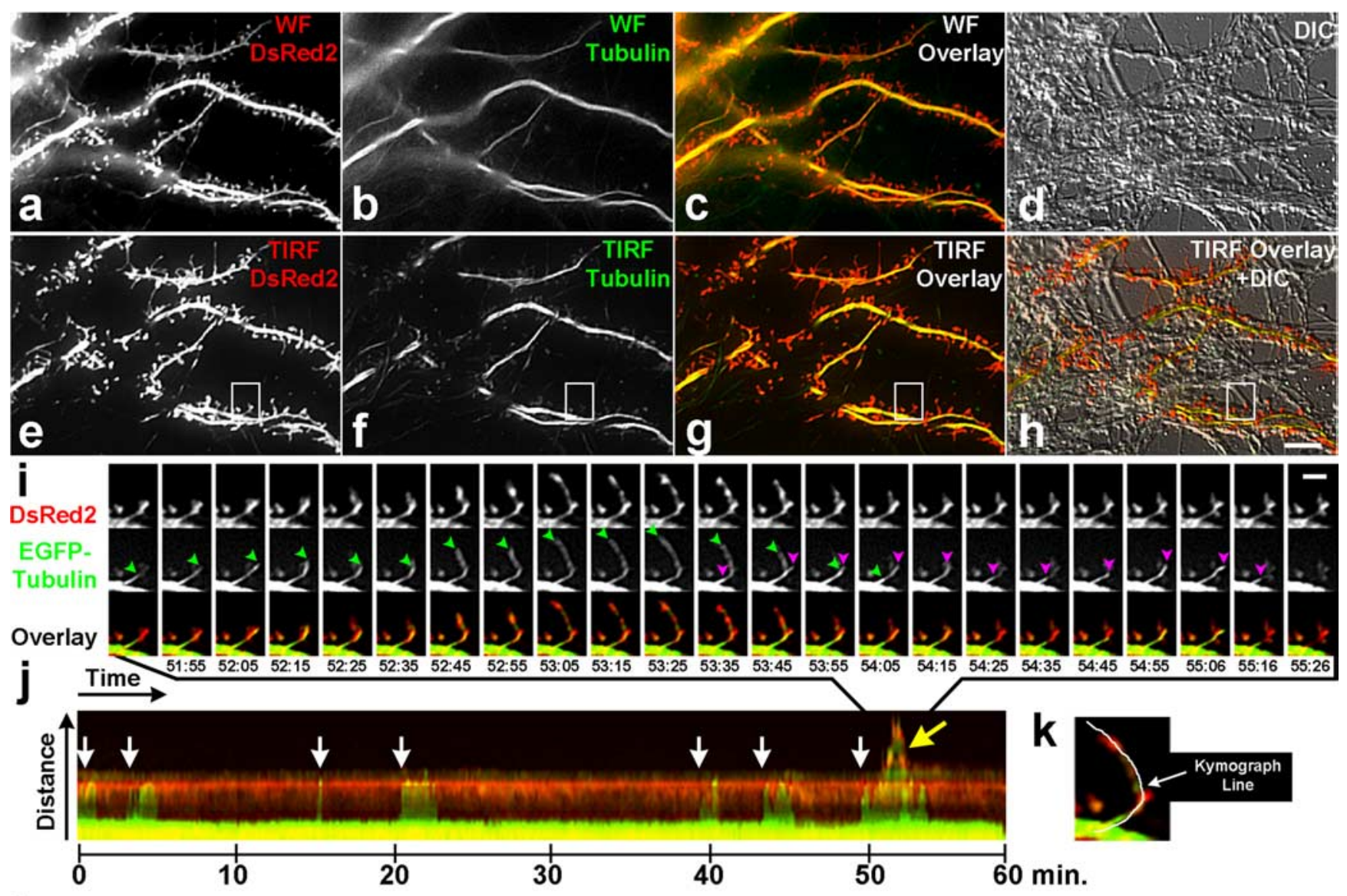

1

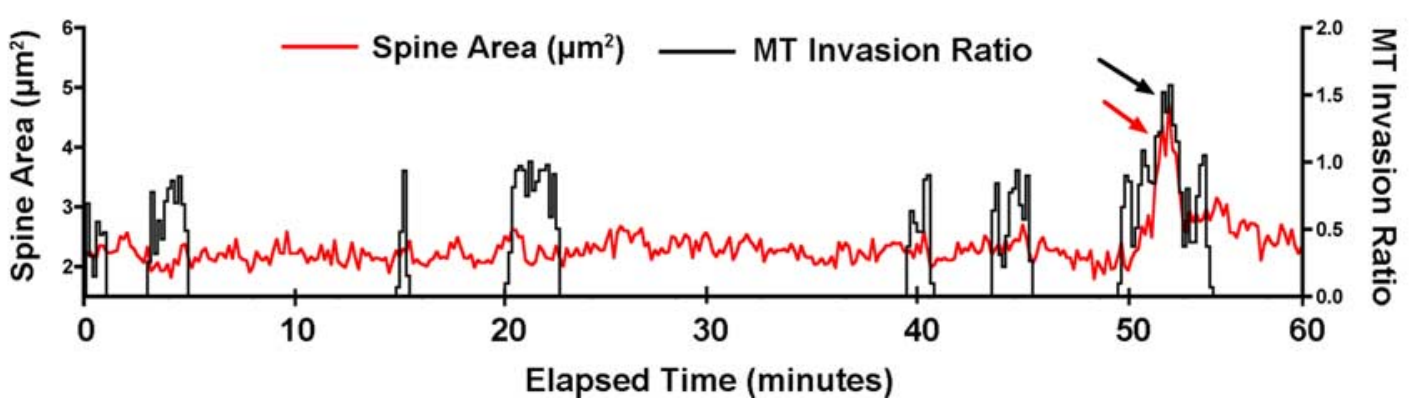

Figure 1. Two microtubules can occupy a spine and induce transient spine head protrusions. Widefield (WF) ( $\boldsymbol{a}-\boldsymbol{c}$ ) and total internal reflection fluorescent (TIRF) (e-g) images of a DIV 20 hippocampal neuron transfected with DsRed2 $(\boldsymbol{a}, \boldsymbol{e})$ and EGFP- $\alpha$-tubulin $(\boldsymbol{b}, \boldsymbol{f})$ at the time of plating. Overlay images of WF $(\boldsymbol{c})$ and TIRF $(\boldsymbol{g})$ images with DsRed2 in red and EGFP-tubulin in green. $\boldsymbol{d}$, DIC image of same region. $\boldsymbol{h}$, DIC image merged with TIRF overlay image $(\boldsymbol{g})$. $\boldsymbol{i}$, Montage of images $(\sim 4 \mathrm{~min})$ from a $1 \mathrm{~h}$ TIRF time-lapse of the spine outlined by the white box (in $\boldsymbol{e}-\boldsymbol{h}$ ). This spine is targeted by two different microtubules during the time interval shown in the montage. $\boldsymbol{i}$, The distal extent of each microtubule is demarcated by green and magenta arrowheads (in the EGFP-tubulin row). The entry of the first microtubule (green arrowheads) coincides with a transient spine head protrusion (tSHP) (52:45-53:55 min). The first microtubule (green arrowheads) depolymerizes (53:35-54:05 $\mathrm{min}$ ) as the second microtubule polymerizes into the spine (53:35-55:06).j, A kymograph constructed from a five pixel wide line drawn through the extent of the spine and tSHP $(\boldsymbol{k})$. The kymograph line $(\boldsymbol{k})$ is linearized and compiled horizontally for each frame (10 sinterval) of the $1 \mathrm{~h}$ time-lapse (361 frames). Time is indicated on the $x$-axis and distance is indicated on the $y$-axis. White arrows in $\boldsymbol{j}$ mark the beginning of separate microtubule excursions (events) into the spine and the tSHP is indicated by the yellow arrow. $I$, Graph plotting changes in spine area for the spine shown in $\boldsymbol{e}-\boldsymbol{k}$ and microtubule invasions into that spine. MT invasion ratios were calculated by taking the ratio of the length of MT invasion into the protrusion divided by the protrusion length (DsRed2 channel). A protrusion ratio of 1.0 indicates the MT extended to the tip of the protrusion. Numbers $>1.0$ indicate the formation of a transient spine head protrusion (tSHP) in which the length to the distal tip of the spine head proper is used as the denominator. Scale bar is $5 \mu \mathrm{m}$ in $\boldsymbol{h}$ and $2 \mu \mathrm{m}$ in the last DsRed 2 frame of $\boldsymbol{i}$.

mental Movie 1, available at www.jneurosci.org as supplemental material). We were able to image neurons with standard widefield microscopy (WFM) but the images were of lower quality with higher background fluorescence (Fig. 1a-c). Furthermore, the much higher level of illumination required to obtain usable WFM images limited our total imaging time to 20-30 min before phototoxic damage to the neurons occurred. Therefore, all livecell imaging was conducted with TIRFM. However, we used
WFM to obtain z-stacks of fixed cultures to detect MTs in protrusions after fixation (see below). All of our studies were performed on cultures that were transfected by electroporation before plating. Because all of the neurons that we imaged expressed the transfected proteins for their entire time in culture (DIV 1263 ) and developed normally, it is unlikely that transfection of EGFP- $\alpha$-tubulin or EB3-EGFP induced spurious cytoskeletal dynamics in our cultures. Surprisingly, we discovered that MTs 
remained dynamic in mature cultures of both hippocampal and cortical neurons (DIV 12-63). However, for quantitative analysis of MT dynamics in spines we imaged EGFP- $\alpha$-tubulin-labeled MTs (and DsRed2) in DIV 20-28 hippocampal neurons at $10 \mathrm{~s}$ intervals for $1 \mathrm{~h}$, resulting in 361 images for analysis of each protrusion.

\section{Microtubules transiently invade dendritic protrusions}

In all types of dendritic protrusions examined (filopodia, stubby spines, thin spines and mushroom-shaped spines) MTs were capable of rapidly extending into and out of the full extent of the protrusion (supplemental Movie 2, available at www.jneurosci. org as supplemental material). Furthermore, in one instance we documented that two MTs could occupy one spine at a time (Fig. $1 i$; supplemental Movie 3, available at www.jneurosci.org as supplemental material). The first MT polymerized (green arrowheads) into the spine at the beginning of the montage (51:44) and continued polymerizing coincident with the formation of a transient spine head protrusion (tSHP) (52:45-53:25) (Fig. 1i). This MT then depolymerizes out of the spine coincident with retraction of the tSHP (53:35-54:05), whereas a second MT (magenta arrowheads) polymerizes into the spine without inducing a tSHP. tSHPs were seen in a subset of spines that were targeted by MTs (6.0\%, 4 of 69 spines, DIV 20-28) and appeared to extend along other neuronal processes near the spine (Fig. $1 h$, boxed region). MT polymerization/depolymerization always accompanied tSHP extension/retraction (Fig. 1j, yellow arrow) indicating an important association of MTs and tSHPs. In fact, in DIV 20-28 cultures tSHPs only occurred when MTs polymerized beyond the spine head (7 of 7 tSHPs from 4 different spines). Furthermore, we did not observe tSHPs in spines that were not targeted by MTs ( 0 of 76 randomly selected untargeted spines). tSHPs were always transient, with an average lifetime of $40 \pm 12 \mathrm{~s}(n=7)$.

Because we used DsRed2 as a marker of cell volume we may have missed tSHPs that were entirely actin-based filopodial protrusions. Indeed, based on our imaging of very mature neurons (DIV 63), that were transfected with mCherry- $\beta$-actin instead of DsRed2, we document transient actin-based protrusions that did not contain MTs (see Fig. 6). Thus, there may be both MT- and f-actin-based extensions from spine heads. Our current imaging technique does not allow us to determine whether MTs perforated the postsynaptic density or whether these protrusions extend into or along the outside of the presumptive presynaptic ending associated with these spines. Nevertheless, the vast majority of spines in our cultures are associated with synaptophysin puncta in both DIV 20-28 untransfected cultures (94.7 $\pm 0.8 \%$, $n=1459$ spines) and DsRed2/EGFP- $\alpha$-tubulin-transfected cultures ( $93.9 \pm 1.3 \%, n=1098$ spines), indicating the presence of a morphological presynaptic ending associated with most spines. Similar values for transfected and nontransfected cultures $(p>$ 0.05 , Student's $t$ test with Welch's correction) also suggests that long-term transfection with DsRed 2 and EGFP- $\alpha$-tubulin does not affect synaptogenesis in our cultures.

To represent, in a single image, the frequency and extent of MT polymerization in protrusions over $1 \mathrm{~h}$ imaging intervals, we compiled kymographs. These kymographs consisted of taking the maximum pixel value from a five pixel-wide line drawn parallel to the long axis of the protrusion for each frame (Fig. $1 k$ ) and arranging them horizontally, yielding images in which the $x$-axis represents time and the $y$-axis represents distance (Fig. 1j). The maximum value within a five pixel-wide line (instead of using a single pixel-wide line) was used to assure that we recorded all MT invasions within each motile spine. The kymograph of this spine shows that MTs enter protrusions by discrete invasions (Fig. $1 j$, white arrows) and polymerize/depolymerize coincident with extension/retraction of a tSHP (Fig. $1 j$, yellow arrow). We measured MT invasions into spines ( $n=69$ spines, 24,909 images) and plotted them graphically (Fig. $2 a$ shows four examples). These four examples show the variability of MT invasion; some spines are targeted frequently, whereas others are targeted rarely or not at all (data not shown). To determine whether MT invasion varied over $1 \mathrm{~h}$ of imaging, we compiled a cumulative graph of MT invasion of spines after binning MT invasions in 10 min intervals for each of the 69 spines (Fig. 2b). This graph shows that the percentage of time a MT is present in a spine does not vary over the $1 \mathrm{~h}$ of imaging, indicating that TIRF imaging does not affect the likelihood of MT invasion into dendritic spines ( $p=0.567$, repeated measures ANOVA and $p=0.189$, post test for linear trend). We did not find significant morphological changes (area, spine length, spine head width) in dendritic spines when MTs invaded these protrusions, except when the invasion resulted in a tSHP (Fig. $1 l$ ). These increases in spine area were always transient, coinciding with the presence of the MT in the spine (Fig. 1l). However, because TIRF imaging does not allow us to image the full extent of the spine in the $z$-axis changes in spine volume may be occurring during MT invasion.

\section{Microtubule kinetics differ in spines versus filopodia}

Overall, we found MTs explored $8.9 \pm 1.0 \%$ of protrusions (spines and filopodia) per hour (104 of 1163 protrusions from 10 independent experiments), indicating only a subset of spines are visited in a given time period. However, this number may be an underestimate of the actual number of protrusions invaded by MTs because TIRF imaging only allows us to image MTs that are within the $\sim 400 \mathrm{~nm}$ distance above the coverslip. To determine the kinetics of MT invasion of dendritic protrusions we quantified MT dynamics in filopodia $(n=23)$ and spines (thin and mushroom-shaped) ( $n=69$ ) from DIV 20-28 hippocampal neurons transfected with EGFP- $\alpha$-tubulin/DsRed2 (Fig. 3). Spines and filopodia were differentiated by strict morphological criteria (see Materials and Methods). All filopodia in which MT invasion was quantified maintained a relatively fixed length $( \pm 0.3 \mu \mathrm{m})$ for the entire hour of imaging (the few filopodia that were dynamic during the $1 \mathrm{~h}$ interval were not quantified). Stubby spines were not quantified because of the difficulty of discerning MT entry into a spine directly apposed to the brightly fluorescent dendritic shaft. Furthermore, we combined the data for thin and mushroom-shaped spines because they were statistically similar.

We quantified a number of different dynamic parameters including: the percentage of time MTs were present in protrusions (Fig. $3 a$ ), the average event time (defined as the cumulative time it takes an individual MT to enter and exit a protrusion) (Fig. 3b), the percentage of time a MT within a protrusion was extending, pausing and retracting (Fig. 3c), the number of both full (extension to the tip of the protrusion) and partial MT invasions per hour (Fig. $3 d$ ), and the average polymerization/depolymerization rates of MTs in protrusions. Only protrusions that were invaded by MTs were quantified. MTs spent a significantly higher percentage of time in spines $(9.9 \pm 1.3 \%)$ compared with filopodia $(5.3 \pm 0.8 \%)$ (Fig. $3 a)$. This higher percentage of time spent in spines may be due to both longer event times $(141 \pm 11 \mathrm{~s}$ for spines vs $86 \pm 9$ s for filopodia) (Fig. $3 b$ ), which were dependent on MTs pausing more in spines ( $42 \pm 3 \%$ of time in spines vs $32 \pm 4 \%$ of time in filopodia) (Fig. $3 c$ ) and the slightly, but not significantly, higher number of invasion per hour $(3.2 \pm 0.5$ 
spines vs $2.6 \pm 0.5$ filopodia) (Fig. $3 d$ ). Furthermore, the likelihood of full MT invasion was similar between spines $(68.4 \%$, $n=212$ events $)$ and filopodia $(69.5 \%, n=$ 59 events), indicating that MTs were just as likely to polymerize to the end of spines as filopodia, although filopodia were considerably longer. Similarly, polymerization (spines, $6.6 \pm 0.2 \mu \mathrm{m} / \mathrm{min}$; filopodia, $6.7 \pm 0.3 \mu \mathrm{m} / \mathrm{min}$ ) and depolymerization (spines, $7.2 \pm 0.2 \mu \mathrm{m} / \mathrm{min}$; filopodia, $7.0 \pm 0.4 \mu \mathrm{m} / \mathrm{min}$ ) rates of MTs in spines and filopodia did not differ from each other or from values documented in newly plated cortical neurons (Dent and Kalil, 2001). These data indicate that MTs target spines slightly more frequently and spend more time per targeting event by pausing more in spines compared with filopodia, resulting in MTs spending a higher overall percentage of time in spines.

Microtubules enter dendritic protrusions from both the proximal and distal dendrite

MTs are polar polymers that polymerize and depolymerize rapidly from their plus end in a process termed dynamic instability (Mitchison and Kirschner, 1984). Because MTs in dendrites are of mixed polarity (arrayed with their plus (dynamic) ends facing both proximal and distal directions (Baas et al., 1988)) we wondered whether MT entry into dendritic protrusions occurred from both the proximal and distal dendrite. Stated differently, could a MT with it's plus end facing distally polymerize into a protrusion/spine (from the proximal dendrite), as well as a MT that was arrayed in the dendrite with it's plus end facing proximally (polymerization into the spine/protrusion from the distal dendrite)? Indeed, we documented that EGFP- $\alpha$-tubulin-labeled MTs can enter protrusions from both the proximal and distal dendrite with live-cell TIRFM (supplemental Fig. 1, supplemental Movie 4, available at www.jneurosci.org as supplemental material). To confirm these results we also labeled the polymerizing ends of MTs with the +TIP protein EB3 (Stepanova et al., 2003). Unlike EGFP- $\alpha$-tubulin, EB3-EGFP only allows the detection of polymerizing MTs, not pausing or depolymerizing MTs, so we could not use these data in our quantifications above. Nevertheless, an advantage of using EB3-EGFP is that MT polymerization within dendritic shafts, as well as dendritic protrusions, can be documented. This allowed us to unequivocally show that MTs were capable of entering protrusions from both proximal and distal dendritic domains (Fig. 4; supplemental Movie 5, available at www.jneurosci.org as supplemental material). Most of the invasions occurred from the proximal dendrite $(84.8 \%)$, whereas fewer occurred from the distal dendrite $(15.2 \%)(n=92$ invasions). These percentages are different from

b
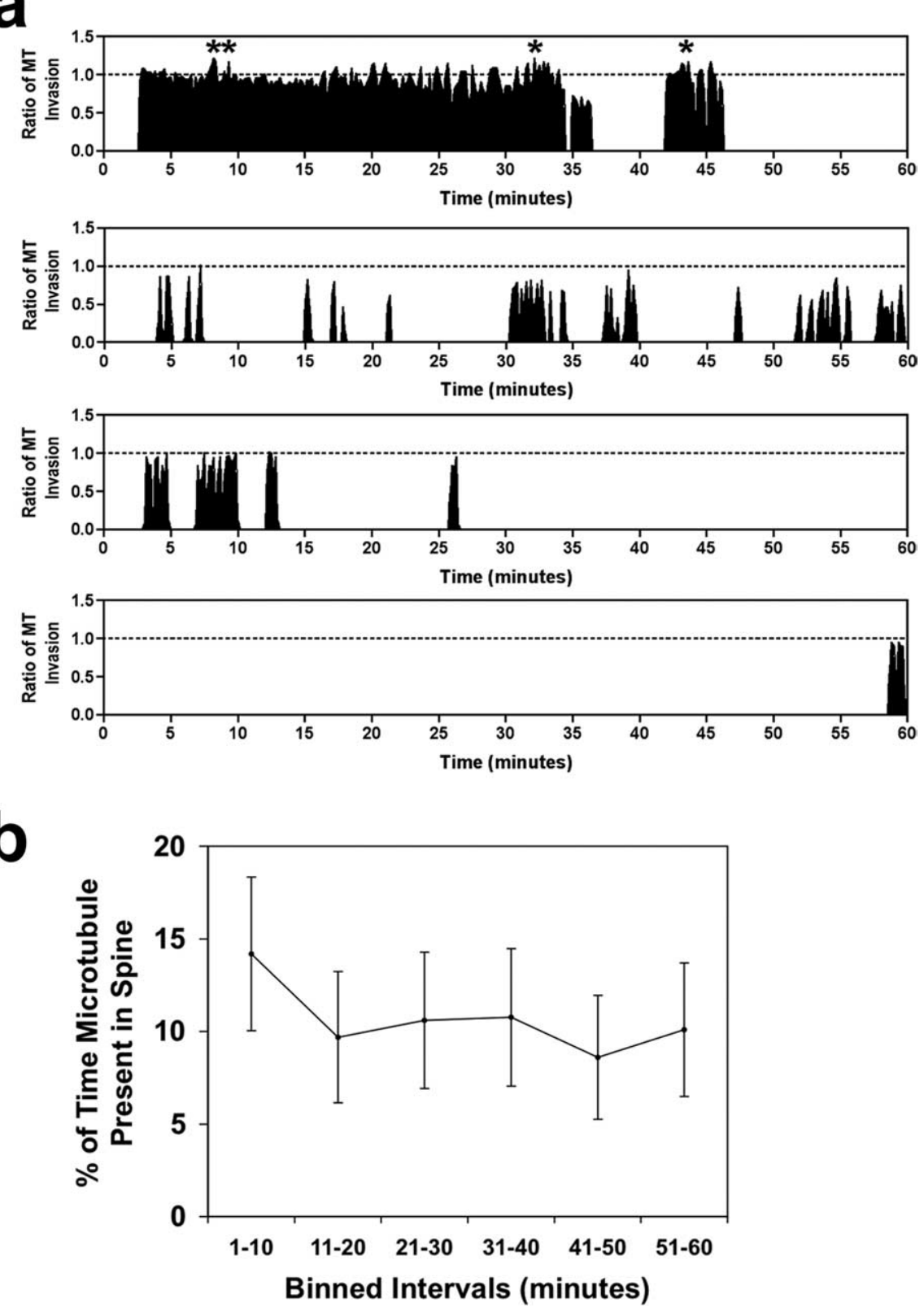

Figure 2. Microtubules invade spines consistently over $1 \mathrm{~h}$ of imaging. $\boldsymbol{a}$, Representative graphs of MT entry into spines as a function of time. The $x$-axis is time $(0-60 \mathrm{~min})$ and the $y$-axis is the ratio of invasion $(0.0-1.0$, except during $\mathrm{tSHPs}$ in which values vary from 1.10 to 1.22 for examples shown here). The asterisks above the invasions in the first graph indicate tSHPs. $\boldsymbol{b}$, Cumulative any of the data points over the $1 \mathrm{~h}$ imaging interval ( $p=0.567$, repeated measures ANOVA) nor a linear trend over time ( $p=$ 0.189 , post test for linear trend).

the percentages of MTs arrayed with plus ends facing away from the cell body or toward the cell body in both hippocampal $(\sim 60 \%$ away/40\% toward) (Baas et al., 1988) and Purkinje ( $\sim 65 \%$ away/ $35 \%$ toward) (Stepanova et al., 2003) dendrites. However, both of these studies found most ( $\sim 95 \%)$ MTs in the distal dendritic regions, at which we focused much of our imaging, faced away from the cell body (Baas et al., 1988; Stepanova et al., 2003), indicating the percentages of MT invasions we documented are in line with the polarity of MTs in the these regions of the dendrite. These data indicate that MTs of either polarity are capable of entering dendritic protrusions.

In addition to polymerizing into and depolymerizing out of 


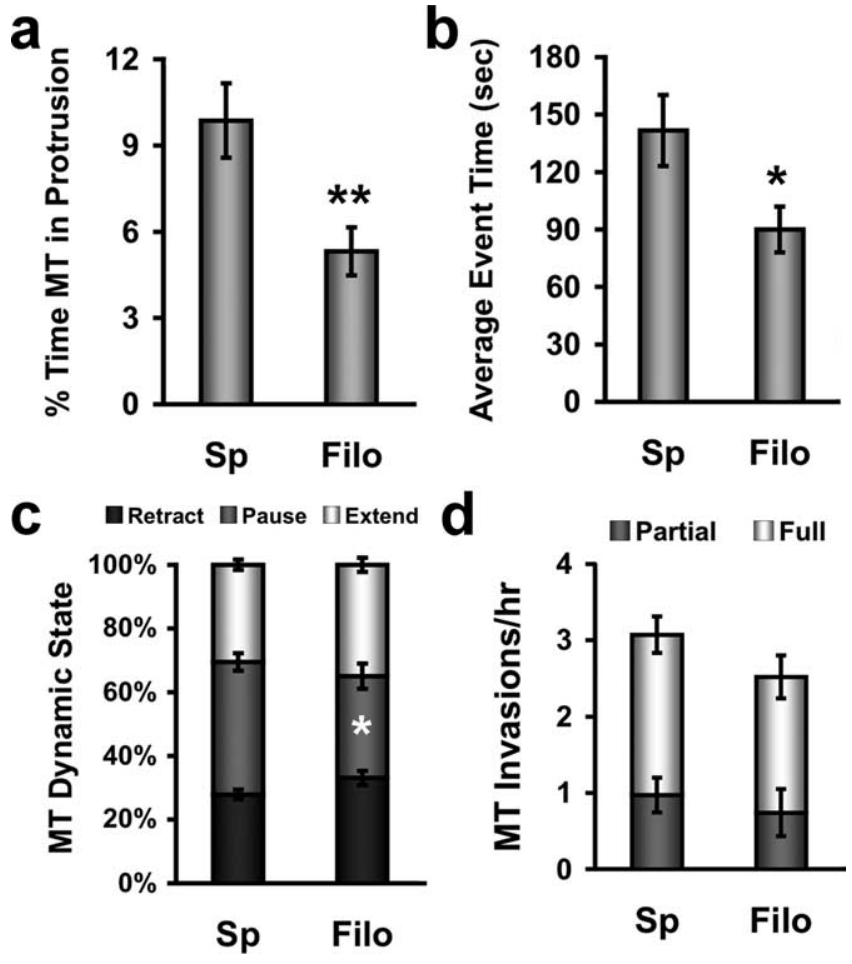

Figure 3. Microtubules remain in spines longer and pause more than in stable filopodia. $\boldsymbol{a}$, Bar graph showing the average percentage of time microtubules are present in protrusions during a $1 \mathrm{~h}$ interval (only spines invaded by MTs were quantified). Microtubules spend a significantly longer time in spines (Sp) than in stable filopodia (Filo) ${ }^{* *} p<0.01$, Student's $t$ test with Welch's correction). $\boldsymbol{b}$, Bar graph showing the average time a microtubule spends in a protrusion for each event (excursion). Microtubules spend a significantly longer amount of time per excursion in spines compared with filopodia $\left(^{*} p<0.05\right.$, Student's $t$ test with Welch's correction). $c$, Stacked bar graph showing the percentage of time microtubules spend retracting, pausing and extending while present in spines and filopodia. Microtubules in spines spend a significantly longer time in the paused state than MTs in filopodia $\left({ }^{*} p<0.05\right.$, Student's t test with Welch's correction). $\boldsymbol{d}$, Bar graph showing the number of partial and full microtubule invasions into spines and filopodia per hour do not differ significantly. ( $n=69$ spines, $n=23$ filopodia, from DIV 20-28 hippocampal neurons).

spines, we documented a single case in which a MT polymerized into a spine followed by a depolymerization from its minus (trailing) end while in a spine (supplemental Fig. 2, supplemental Movie 6, available at www.jneurosci.org as supplemental material). This observation indicates that relatively short MTs are capable of polymerizing into spines. We never observed a MT originating (nucleating) in a spine and polymerizing out of it into the dendrite shaft, as has been suggested by others (van Rossum and Hanisch, 1999). However, this type of MT behavior is formally possible.

\section{EGFP-labeled and endogenous microtubules target spines with morphological synapses}

Although our DIV 20-28 cultures were mature and likely contained presynaptic endings adjacent to spines we wanted to establish whether MTs were capable of entering spines containing a morphological synapse. Furthermore, we wanted to determine whether we could image MTs in spines away from the coverslip interface with standard wide-field imaging to determine whether MT invasion of dendritic protrusions only occurred in spines adjacent to the coverslip. First, we fixed DIV 20-28 cultures previously transfected with EGFP- $\alpha$-tubulin/DsRed2, stained them for presynaptic endings with anti-synaptophysin and imaged them by taking z-series stacks ( 10 images, $0.2 \mu \mathrm{m}$ distance be- tween images, $100 \times / 1.49$ NA objective) with wide field imaging (Fig. 5a,b). MTs in EGFP- $\alpha$-tubulin/DsRed2-transfected neurons (not directly on the coverslip) entered spines and targeted morphological synapses. These data are consistent with another study that used confocal imaging in fixed cultures and documented the presence of MTs in spines (Gu et al., 2008). From our live-cell analysis we determined MTs usually enter spines at nonright angles relative to the dendrite shaft, as we have shown for MTs entering axon branches from the parent axon (Dent et al., 1999, 2003; Kalil et al., 2000; Dent and Kalil, 2001). MTs predominantly targeted spines at an obtuse angle, relative to the dendrite shaft. Therefore, in fixed neurons we could determine whether MTs targeted spines from proximal vs distal dendrites by the angle they formed in the spine neck, relative to the dendrite shaft (Fig. $5 a, b)$. We found that most spines $(90 \%, n=10)$ in transfected and fixed neurons were targeted from the proximal dendrite (spines targeted by MTs at right angles were disregarded). This value is similar to the percentage of spines targeted from the proximal dendrite $(85 \%)$ in our live-cell imaging studies (see above).

However, EGFP- $\alpha$-tubulin-containing MTs may exhibit aberrant MT behavior. Therefore, as a second approach we determined whether endogenous MTs from untransfected neurons enter dendritic spines. We fixed untransfected DIV 20-28 hippocampal cultures and stained them for endogenous, newly polymerized MTs (anti-tyrosinated MTs), dendritic spines (phalloidin to label high concentrations of f-actin in spines) and presynaptic endings (anti-synaptophysin). We imaged these labeled cultures in TIRFM to distinguish endogenous MT invasion of spines from the highly tubulin-labeled dendrites and axons in the culture. Endogenous MTs extended into dendritic protrusions from both proximal $(86 \%)$ and distal (14\%) dendrites as well ( $n=22$ spines) (Fig. $5 c, d)$. Thus, by live-cell imaging of transfected neurons with TIRFM, fixed-cell imaging of transfected neurons with wide-field microscopy and fixed cellimaging of endogenous MTs with TIRFM we consistently found that $\sim 85 \%$ of protrusions were targeted from the proximal dendrite and $\sim 15 \%$ of protrusions were targeted from the distal dendrite. It should be noted that we fixed all of our cultures in a fixative that preserves dynamic MTs and f-actin and stained them for tyrosinated-tubulin to detect newly polymerized/dynamic MTs (Dent et al., 2007).

In fixed cultures endogenous MTs targeted spines at a similar frequency to EGFP-labeled MTs. Overall, $1.1 \pm 0.3 \%$ of spines contained endogenous MTs ( $n=3477$ spines $)$ and $0.9 \pm 0.3 \%$ of spines contained EGFP- $\alpha$-tubulin-labeled MTs $(n=1098$ spines) ( $p>0.05$, Student's $t$ test). Endogenous MTs can extend to the tips of spines as well. Importantly, the percentage of spines containing MTs in fixed cultures is in accordance with another study by Zheng and colleagues (Gu et al., 2008) in which confocal imaging of living rat hippocampal neurons was used to show $1-2 \%$ of spines contained MTs. Together with our data, these results indicate similar MT behavior in both mouse (this study) and rat hippocampal neurons (Gu et al., 2008), regardless of imaging technique used (TIRFM, widefield or confocal). These data also indicate that EGFP- $\alpha$-tubulin-labeled MTs do not induce spurious MT behavior in mature dendrites. Additionally, MTs are present in a very small fraction of dendritic protrusions in fixed specimens, consistent with their extremely rare incidence in electron micrographs of spines in culture or in hippocampus (Gray et al., 1982; Chicurel and Harris, 1992; Fiala et al., 2003). However, we discovered that $8.9 \%$ of spines are targeted per hour (see above), indicating many spines on a neuron may be targeted 
a

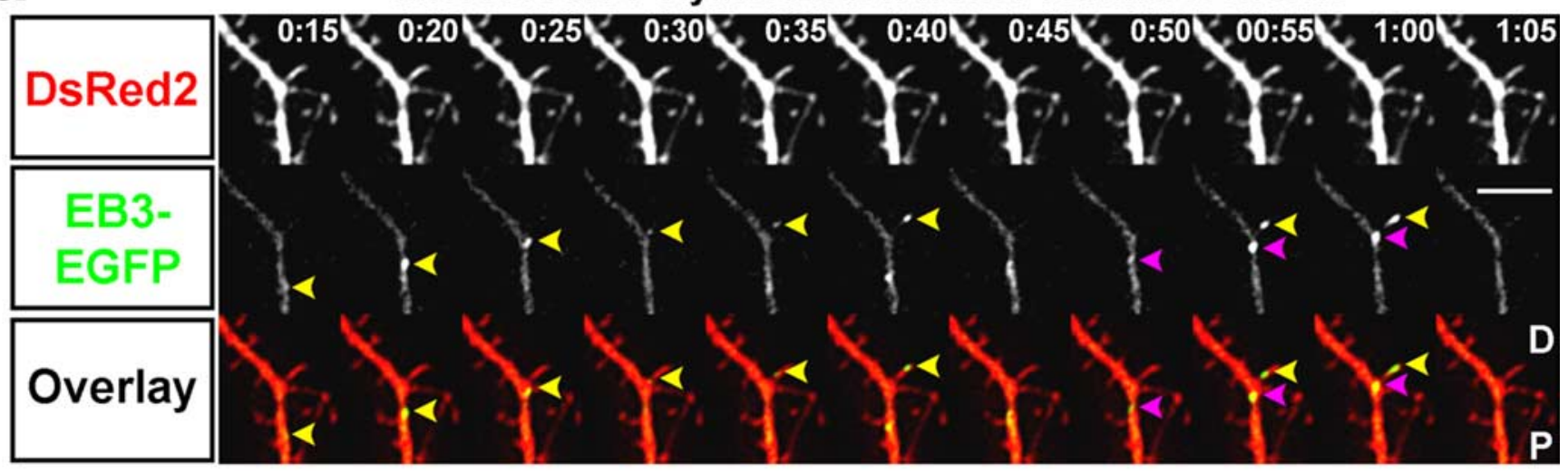

b

Microtubule Polymerization from Distal Dendrite

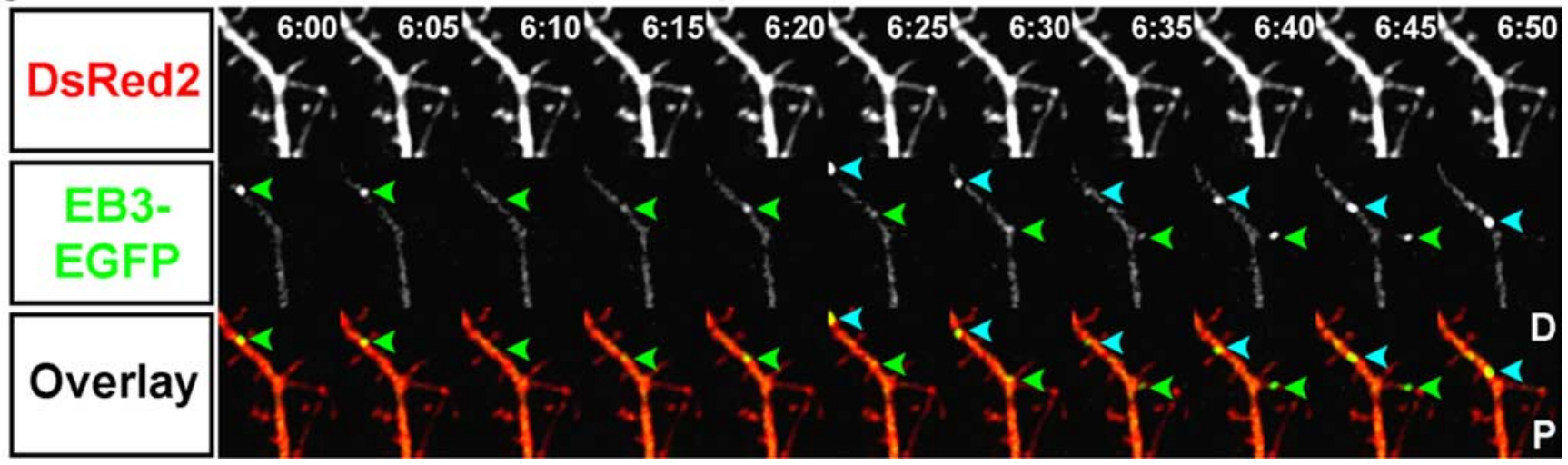

Figure 4. Microtubules (EB3-labeled) enter spines from both the proximal and distal dendrite in a cortical neuron (DIV 12). $\boldsymbol{a}$, An example of anterograde MT polymerization into a dendritic protrusion. An EB3-EGFP punctum (yellow arrowhead) starts to polymerize in the proximal dendritic shaft $(P)(0: 15-0: 25$ min), turns into the protrusion ( $0: 30$ min), and polymerizes to the tip of the protrusion (0:40 min). Another MT (magenta arrowhead) polymerizes in the dendrite shaft proximal to the first MT but does not enter the protrusion (0:50 -1:05 min). The first MT polymerizes again while in the protrusion (0:55-1:00 min). $\boldsymbol{b}$, An example of retrograde MT polymerization in the same dendritic region as $\boldsymbol{a}$. An EB3-EGFP punctum (green arrowhead) begins to polymerize in the distal dendrite shaft (D) (6:00 - 6:25 min), turns into a different protrusion (6:30 min) and polymerizes toward the spine head (6:30 - 6:40 min) but does not reach it (6:45 min). A second MT (cyan arrowhead) polymerizes retrogradely entirely within the dendrite shaft. Time is in minutes:seconds. Scale bar, $5 \mu \mathrm{m}$.

by MTs over a period of a day. Our live cell imaging data correspond very well with our fixed cell data. We discovered that $8.9 \%$ of spines are targeted by MTs per hour and in these spines MTs are present $9.9 \%$ of the time (Fig. 3a). Thus, if a single frame of a $1 \mathrm{~h}$ time-lapse was chosen at random the likelihood of finding a MT in a spine would be $0.88 \%$, which compares favorably with our results from transfected and fixed cultures (0.9\%).

Microtubules remain dynamic throughout the life of the neuron

Surprisingly, we discovered that even in very mature cultures (DIV 63) MTs were still very dynamic (Fig. 6; supplemental Movie 7, available at www.jneurosci.org as supplemental material). We labeled these cultures with EB3-EGFP, a protein that specifically binds to the polymerizing ends of MTs (Stepanova et al., 2003). We discovered that in very mature cultures (DIV 63) MTs were capable of rapid polymerizing anterogradely and retrogradely throughout dendritic shafts ( $n=5$ neurons) (Fig. 6b,c, arrows point to EB3 puncta; supplemental Movie 7, available at www.jneurosci.org as supplemental material) and axons (data not shown), as shown previously in younger hippocampal (DIV 2-6) and Purkinje (DIV 10-17) cell cultures (Stepanova et al., 2003). We documented EB3-labeled MTs extending into spines in these very mature hippocampal cultures as well (Fig. $6 a-c$, boxed region; supplemental Movie 8, available at www.jneurosci. org as supplemental material). tSHPs formed coincidently with EB3-labeled MTs (Fig. 6d; supplemental Movie 8, available at www.jneurosci.org as supplemental material), like EGFP- $\alpha$ tubulin-labeled MTs (Fig. 1i; supplemental Movie 3, available at www.jneurosci.org as supplemental material). Interestingly, a mushroom-shaped spine from this hippocampal neuron showed prominent increases in actin signal coincident with MT invasion (Fig. 6d,e; supplemental Movie 8, available at www.jneurosci.org as supplemental material), suggesting that signaling between these two polymer systems may occur in spines, as shown previously in axonal filopodia (Dent and Kalil, 2001; Schaefer et al., 2002; Zhou et al., 2002; Dent et al., 2007). These increases in actin signal and formation of transient filopodia protruding from the spine head (see mCh-actin frames 1:31:10-1:32:30 h) indicate that $\mathrm{tSHPs}$ can occur without MTs (Fig. $6 d$ ). However, many of the protrusions from these spines contain prominent EB3 label without detectable actin label (Fig. $6 d$, yellow arrowheads; supplemental Movie 8, available at www.jneurosci.org as supplemental material), indicating MTs may be capable of inducing tSHPs independently of actin polymerization, as has been demonstrated in axonal growth cones (Goldberg and Burmeister, 1992). Quantification of $47 \mathrm{tSHPs}$ from the spine head shown in Figure $6 d$ indicated that 55\% (26 of 47) were MT-based without any accompanying actin polymerization, 19\% (9 of 47) were MTbased with actin polymerization into the $\mathrm{tSHP}$ after MT polymer- 

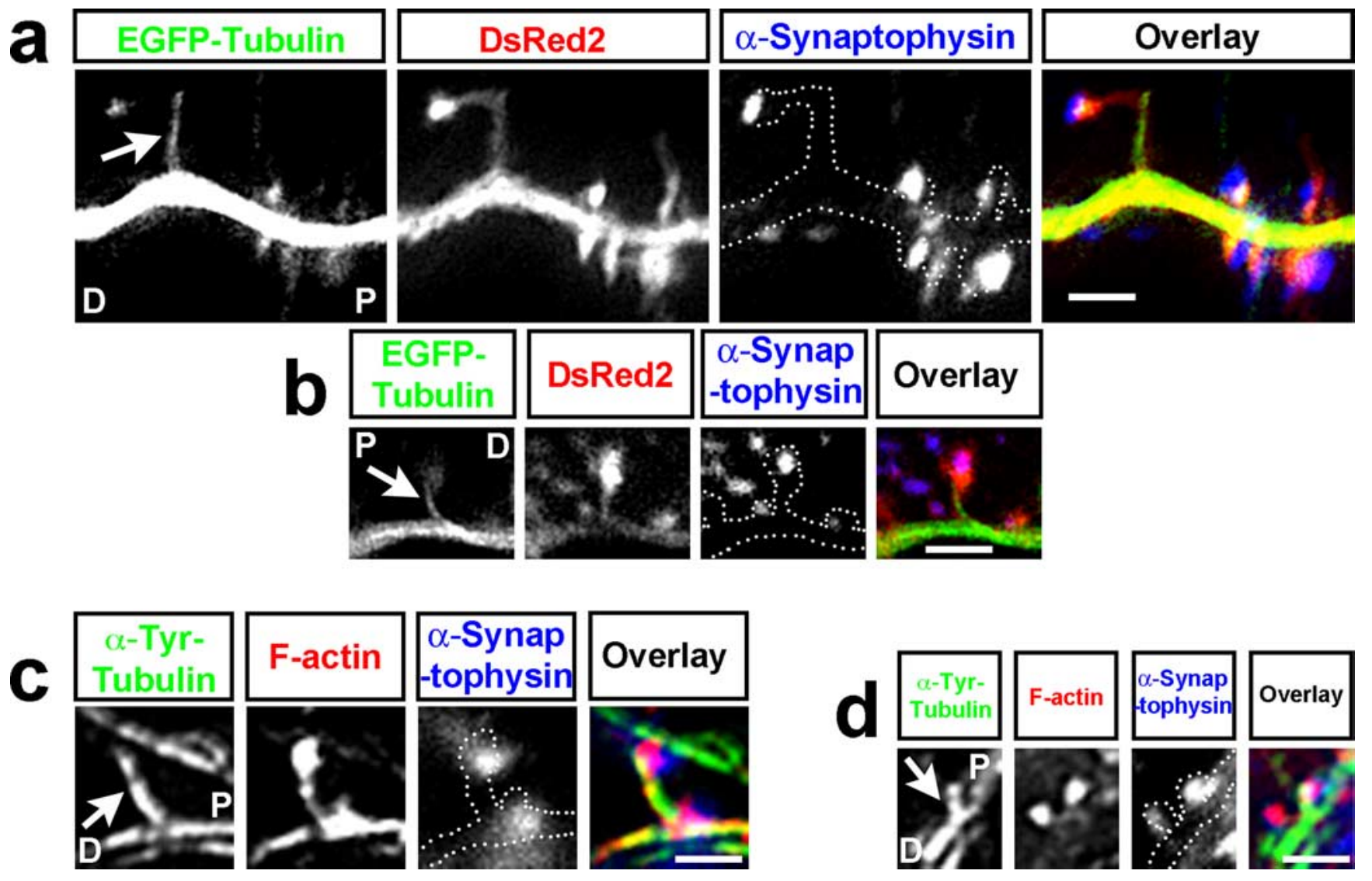

Figure 5. EGFP-labeled and endogenous microtubules target spines from both proximal and distal dendritic regions. $\boldsymbol{a}, \boldsymbol{b}$, Two examples of 20 DIV hippocampal neurons transfected with EGFP-tubulin (green in overlay) and DsRed2 (red in overlay), fixed and stained with an antibody to synaptophysin (blue in overlay), and imaged with wide field microscopy. The DsRed2 image was traced and the tracing was added to the anti-synaptophysin panels to show where the presynaptic endings were in relation to the dendrite. In $\boldsymbol{a}$, the microtubule is entering the spine from the proximal dendrite (P), whereas the microtubule in $\boldsymbol{b}$ is entering from the distal dendrite (D). $\boldsymbol{c}, \boldsymbol{d}$, Two examples of spines from untransfected 20 DIV hippocampal neurons that were labeled with an antibody to tyrosinated tubulin (to label dynamic MTs; green in overlay image), phalloidin (to stain filamentous actin; red in overlay image), and an antibody to synaptophysin (blue in overlay image) and imaged in TIRFM. In $\boldsymbol{c}$, the microtubule is entering the spine from the proximal dendrite (P), whereas the microtubule in $\boldsymbol{d}$ is entering from the distal dendrite (D). Arrows in $\boldsymbol{a}-\boldsymbol{d}$ point to microtubules that are present in spines at the time of fixation. Scale bars: $\boldsymbol{a}-\boldsymbol{d}, 2 \mu \mathrm{m}$.

ization, 9\% (4 of 47) were formed by coincident MT and actin polymerization and $17 \%$ ( 8 of 47 ) were actin-based with no MT polymerization. Based on their infrequent and transient nature further study will be required to determine whether MT-based $\mathrm{tSHPs}$ require $\mathrm{f}$-actin to form.

MT invasion of dendritic protrusions is regulated by activity As a first step toward understanding the functional significance of MT invasion of dendritic spines we experimentally manipulated cultures by stimulating activity with high $\mathrm{KCl}$. We used a regimen of perfusion of $75 \mathrm{~mm} \mathrm{KCl} 3$ times for $3 \mathrm{~min}$, separated by $10 \mathrm{~min}$ rest intervals (Fig. 7a); an established technique to elevate activity in cultured neurons globally but transiently (Wu et al., 2001; Li et al., 2004). We then fixed the neurons after $30 \mathrm{~min}$ and quantified the percentage of protrusions containing a $\mathrm{MT}$. $\mathrm{KCl}$ treatment resulted in a significant increase of protrusions containing a MT from $0.74 \pm 0.30 \%$ ( $n=15$ neurons/1960 protrusions) to $3.05 \pm$ $0.53 \%$ ( $n=13$ neurons $/ 1818$ protrusions), an increase of $>400 \%$ (Fig. $7 b$ ). Conversely, when we globally inhibited activity in the cultures by including $1 \mu \mathrm{M}$ tetrodotoxin (TTX) in the perfusate we decreased the percentage of protrusions containing a MT from $0.74 \pm 0.30 \%$ to $0.42 \pm 0.22 \%$ ( $n=11$ neurons/1982 protrusions), although this decrease was not significantly different from controls (Fig. 7b). Furthermore, by including TTX in all solutions while stimulating with $\mathrm{KCl}$ (Fig. $7 b$ ) we abolished the increase in $\mathrm{MT}$ invasion produced by $\mathrm{KCl}$, resulting in $1.05 \pm$ $0.52 \%$ ( $n=12$ neurons/1364 protrusions) protrusions invaded by MTs (Fig. 7b). This value was not significantly different from control or TTX-treated cultures $(p>0.05$, Kruskal-Wallis test with Dunn's post hoc tests). These data indicate that global activation of synaptic activity increases the proportion of spines containing MTs and this increase in MT invasion can be inhibited by TTX.

To understand the dynamic aspects of MT entry into dendritic protrusions after $\mathrm{KCl}$ treatment we imaged EGFP- $\alpha$-tubulin and DsRed 2 transfected neurons before and after $\mathrm{KCl}$ application. We used a similar protocol to the one used in fixed cultures (above) and compared MT invasion in the 20 min interval before the first $\mathrm{KCl}$ treatment and the $20 \mathrm{~min}$ interval after the final $10 \mathrm{~min}$ rest period after the third $\mathrm{KCl}$ treatment (Fig. $7 c, d$ ). We quantified these results by determining the percentage of time MTs remained in protrusions (Fig. 7e), the average MT event time (Fig. $7 f$ ) and the number of MT invasions (Fig. $7 g$ ), before and after $\mathrm{KCl}$ treatment. When we compared the mean values for all of these quantitative measurements we found the percentage of time MTs spent in protrusions increased from $16 \pm 6 \%$ before $\mathrm{KCl}$ to $46 \pm 7 \%$ after $\mathrm{KCl}$ (Fig. $7 e$ ) $(n=34$ protrusions for all measurements in Fig. $7 e-g$ ). Also, each MT invasion (event time) persisted for a longer period of time $(183 \pm 67 \mathrm{~s}$ before $\mathrm{KCl}$, $468 \pm 83 \mathrm{~s}$ after) (Fig. $7 f$ ) and the number of invasions doubled 

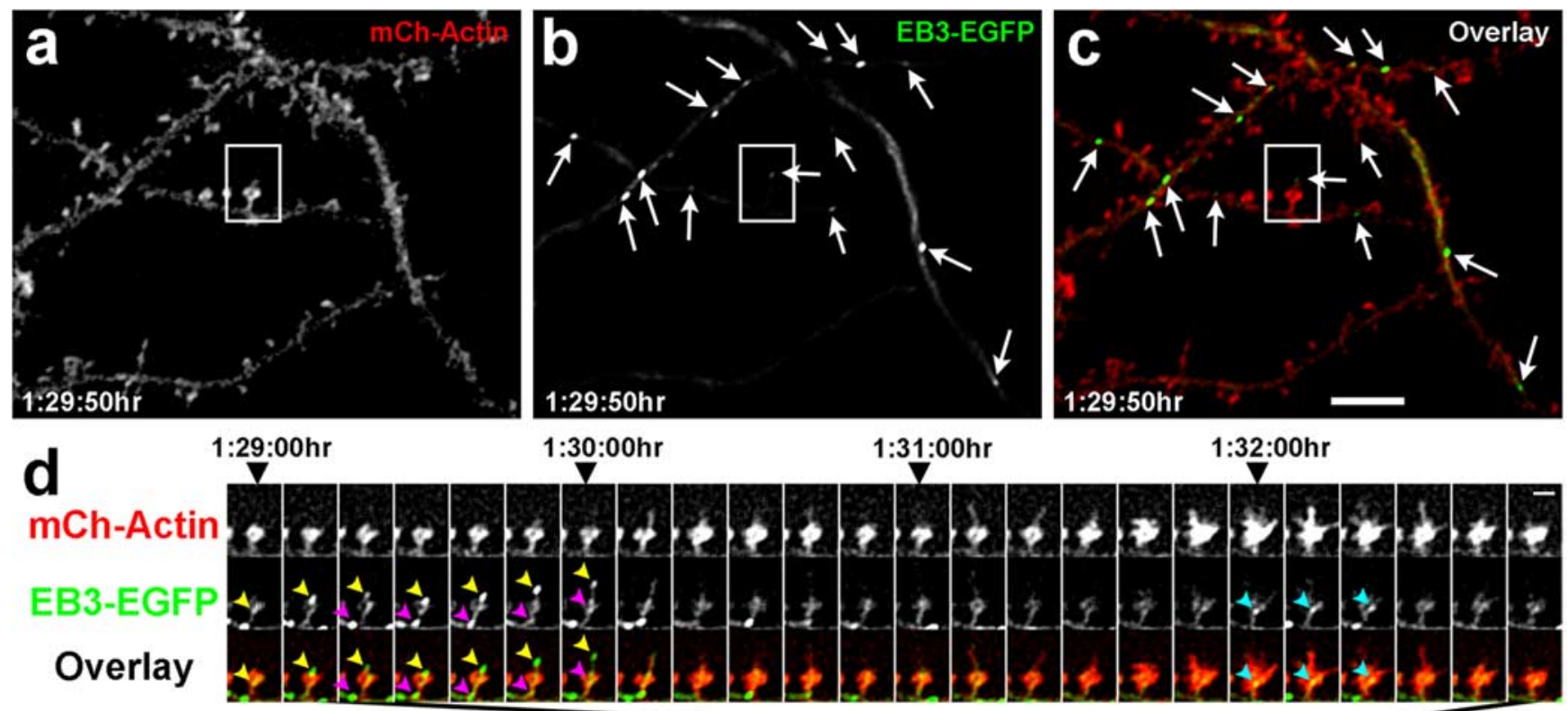

$1: 30: 00 \mathrm{hr}$

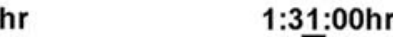

\section{$1: 32: 00 \mathrm{hr}$}

7

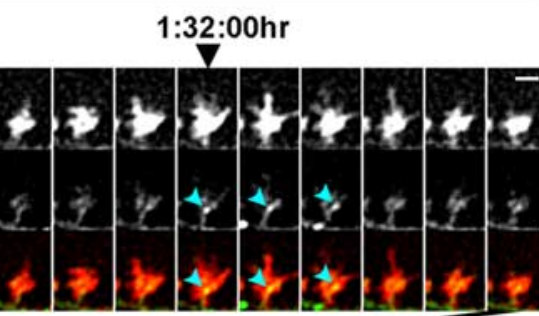

\section{kymograph}

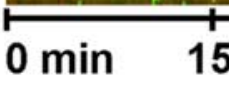

15

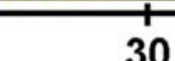

30

45

60

75

$90^{\wedge}$

105

120
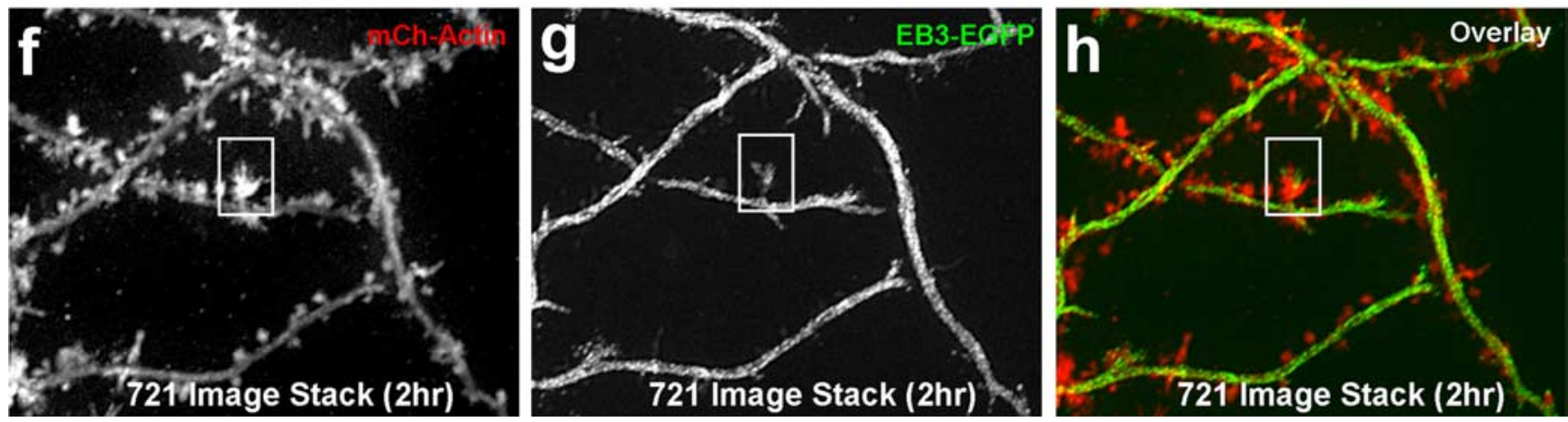

Figure 6. Microtubules remain dynamic throughout the life of the neuron and continue to invade dendritic spines. Fluorescent images of a DIV 63 hippocampal neuron transfected with $m$ Cherry- $\beta$-actin $(\boldsymbol{a})$ and EB3-EGFP $(\boldsymbol{b})$ at the time of plating. Arrows in $\boldsymbol{b}$ and $\boldsymbol{c}$ point to EB3 puncta (polymerizing MTs). $\boldsymbol{c}$, An overlay image of the mCherry- $\beta$-actin (red) and EB3-EGFP (green) images. $\boldsymbol{d}$, A montage of a single spine (boxed in $\boldsymbol{a}-\boldsymbol{c}$ ) from 1:29:00 to 1:32:50 $\mathrm{h}$ in the $2 \mathrm{~h}$ sequence. One MT polymerizes into the spine (yellow arrowheads point to an EB3-EGFP punctum in both the EB3-EGFP and overlay rows) in association with a tSHP and extends to its tip. Another MT (magenta arrowheads point to a second EB3-EGFP punctum) polymerizes into the spine behind the first MT but only extends to the spine head. A third MT (cyan arrowheads) extends into the head of the spine (1:32:00-1:32:20) during the time the actin becomes markedly dynamic (1:31:10 -1:32:40), forming transient filopodia and lamellipodia. $e$, Kymograph of the entire $2 \mathrm{~h}$ sequence showing no MT activity during the first hour but multiple MT excursions (short green lines) interspersed by dramatic actin-based protrusions (black arrowheads) during the second hour. $\boldsymbol{f}-\boldsymbol{h}$, Same regions as in $\boldsymbol{a}$ - $\boldsymbol{c}$ except that these images are composites of all 721 images in the $2 \mathrm{~h}$ time lapse stacked into one image to show movement of actin and EB3. Note that there is so much activity of MTs in the dendrite shafts that they appear white, whereas a single frame shows only bright dots with some background of free $\mathrm{EB} 3(\boldsymbol{b})$. Scale bars: (in $\boldsymbol{c}) \boldsymbol{a}-\boldsymbol{c}, \boldsymbol{f}-\boldsymbol{h}, 5 \mu \mathrm{m}$; (in $\boldsymbol{d}$, last mCh-actin image) $\boldsymbol{d}, \boldsymbol{e}, 2 \mu \mathrm{m}$.

from $0.44 \pm 0.13$ to $0.97 \pm 0.14$ invasions per $20 \mathrm{~min}$ (Fig. $7 g$ ). All measurements made were statistically significant when the means before and after $\mathrm{KCl}$ treatment were compared. Interestingly, we discovered that virtually all protrusions $(n=21$ of 22$)$ that exhibited MT invasion after $\mathrm{KCl}$ treatment had no activity before $\mathrm{KCl}$ treatment (Fig. $7 c$ shows one example) and virtually all protrusions $(n=11$ of 12$)$ that exhibited MT invasion before $\mathrm{KCl}$ treatment did not exhibit any MT invasions after $\mathrm{KCl}$ treatment (Fig. $7 d$ shows one example). A subset of protrusions changed spine type, i.e., filopodia to thin spine or thin spine to mushroom-shaped spine $(13 \%, 4$ of 30$)$. Importantly, the spines that changed type only had MT invasion after $\mathrm{KCl}$ treatment (see Fig. $7 c$ for example). Finally, $\mathrm{KCl}$ induced a significant increase in area for all protrusions, as documented previously (Wu et al.,
2001). This increase in protrusion size, from $2.15 \pm 0.20 \mu \mathrm{m}^{2}$ to $2.72 \pm 0.28 \mu \mathrm{m}^{2}(p<0.0001$, Wilcoxon signed rank test, $n=$ 30 ), occurred whether or not MT invasion took place before or after $\mathrm{KCl}$ treatment, indicating the enlargement was probably not due to MT invasion directly.

\section{Discussion}

Our findings suggest that dendritic MTs are dynamic and can rapidly and transiently polymerize into dendritic protrusions throughout the life of cortical and hippocampal neurons. Interestingly, MTs can penetrate the entire extent of the spine and even induce transient spine head protrusions (tSHPs). Although MTs were only documented in $\sim 1 \%$ of dendritic protrusions in fixed cultures (DIV 20-28) under basal conditions, it is likely that 

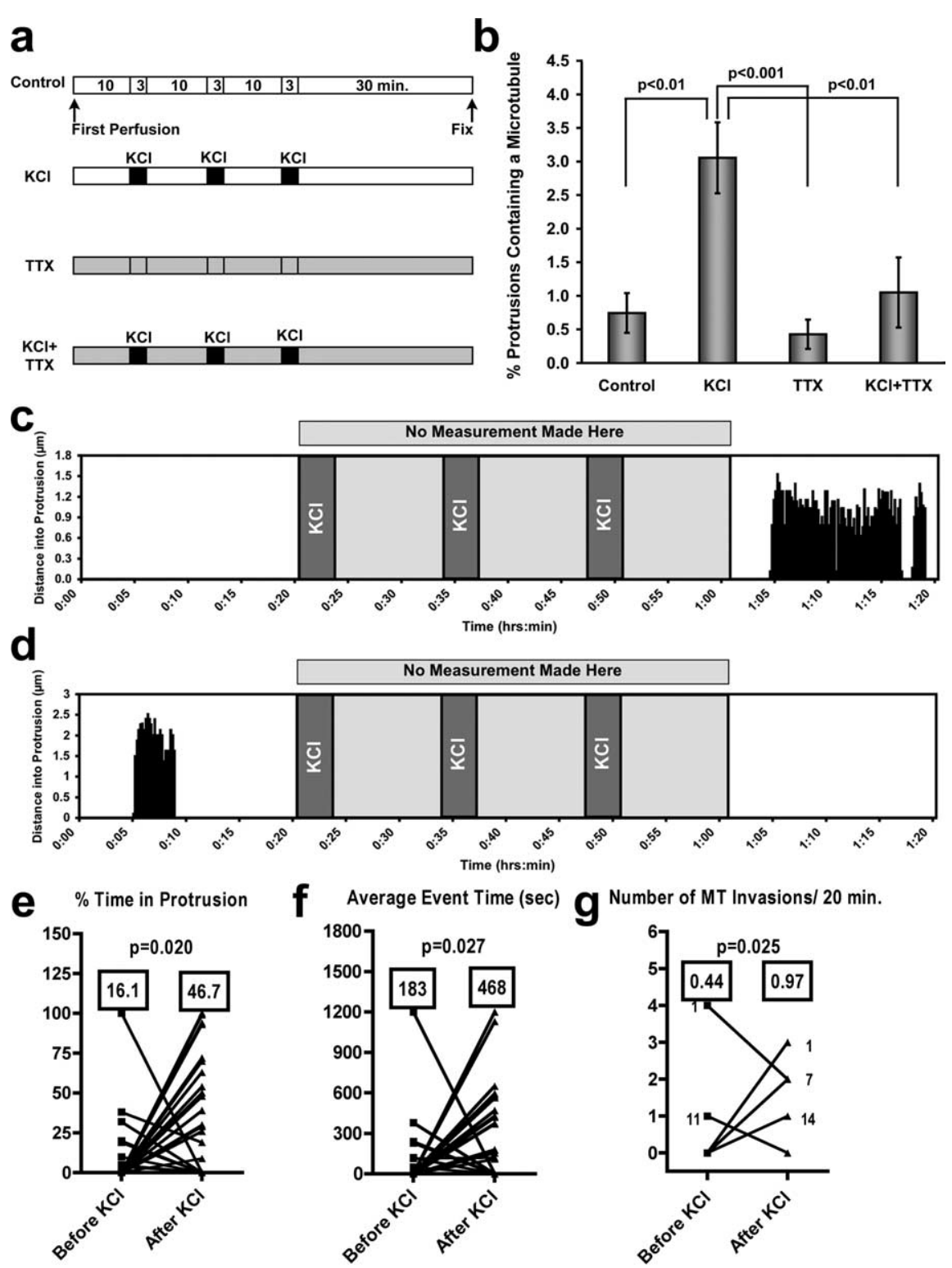

Figure 7. Global activity increases microtubule invasions of dendritic protrusions. $\boldsymbol{a}$, Diagram depicting the perfusion protocols used for $75 \mathrm{~mm} \mathrm{KCl} \mathrm{stimulation} \mathrm{of} \mathrm{global} \mathrm{neuronal} \mathrm{activity} \mathrm{in} \mathrm{DIV} 21$ hippocampal cultures. TTX was included at $1 \mu \mathrm{m}$. $\boldsymbol{b}, \mathrm{Graph}$ of the percentage of protrusions containing a MT at the time of fixation. $\mathrm{KCl}$ treatment induced a substantial increase in the percentage of protrusions containing a MT. TTX addition to the perfusate abolished this increase. $p$ values are shown (KruskalWallis test with Dunn's post hoc tests). Control, TTX and KCI+TTX were not significantly different $(p>0.05)$. c, d, Two examples from 34 protrusions (from hippocampal neurons transfected with EGFP- $\alpha$-tubulin and DsRed2) that were imaged before, during, and after three consecutive $75 \mathrm{~mm} \mathrm{KCl}$ treatments. One graph (c) shows an example of one without MT invasion before $\mathrm{KCl}$ application and substantial MT invasion after, whereas the second graph (d) shows MT invasion before KCl application and no MT invasion after. $\boldsymbol{e}-\boldsymbol{g}$, Graphs quantifying the population of protrusions exhibiting either MT invasion before or after $\mathrm{KCl}$ treatment (all $p$ values computed with Student's $t$ test). Protrusions that showed no MT invasion before or after KCl were not quantified. $\boldsymbol{e}$, MTs are present in protrusions for a significantly higher percentage of time ( $p=0.020$ ) after KCl treatment (means are shown in boxes). $\boldsymbol{f}$, The average time a MT spends in a protrusion during one polymerization/depolymerization event is significantly longer after $\mathrm{KCl}$ treatment $(p=0.027)$. $\boldsymbol{g}$, The number of MT invasions increased significantly after $\mathrm{KCl}$ treatment $(p=0.025)$. All statistics for $\boldsymbol{e}-\boldsymbol{g}$ were calculated with the two-tailed Wilcoxon signed rank test.

many spines are targeted over relatively short periods of time. Indeed, our $1 \mathrm{~h}$ time-lapse imaging of MTs at frequent intervals (10 s) showed that $\sim 9 \%$ of protrusions (spines and stable filopodia) are targeted by MTs. Thus, over a period of a day it is possible that all spines on a dendrite could be targeted by MTs. However, MT invasion of spines is likely to serve specific functions and is therefore not a random event. Indeed, we found that increasing activity substantially increased the number of protru- sions that were invaded by MTs and the time MTs remained in those protrusions, while decreasing activity induced an opposite trend.

\section{Why are spines thought to be devoid of MTs?}

Our results are surprising based on the many light microscopic studies and hundreds of electron microscopic studies that have not documented the presence of MTs in spines. However, there have been a handful of studies that have documented MTs in spines. The first studies that showed MTs in dendritic spines are those by Westrum and Gray (Westrum and Gray, 1976; Westrum et al., 1980, 1983; Gray et al., 1982). However, the occurrence of MTs in spines was dependent on dissection of tissue in up to $20 \%$ bovine serum albumin. This technique was, for good reason, dismissed by many researchers. More recent work showed MTs in complex, branched spines of the CA3 region of hippocampus that contained greater than four heads (Chicurel and Harris, 1992), indicating MT entry only under unusual circumstances. Moreover, a recent study from the same lab showed that the presence of MTs in dendritic spines was likely to be an artifact of warming hippocampal slices and was not documented in freshly perfused tissue (Fiala et al., 2003). These findings at the EM level were confirmed with light microscopy by imaging EGFP-MAP2b and -MAP2c with time-lapse fluorescent microscopy (Kaech et al., 2001). It is unclear why these investigators failed to detect MTs in spines (or much dynamic MAP2 movement) after imaging for several hours. One possibility is that overexpression of MAP2 stabilized MTs. However, this group imaged hippocampal slices from EGFP-MAP2cexpressing mice that did not exhibit any morphological or behavioral abnormalities, making this argument less convincing.

We suggest that the failure to detect MTs in spines can be explained by their inherently dynamic nature. In preparation of samples for electron microscopy MTs are not usually stabilized against depolymerization during fixation. As we show here in mature neurons and have documented in immature neurons (Dent and Kalil, 2001; Dent et al., 2004, 2007), MTs are extremely dynamic polymers that are exquisitely sensitive to perturbation. Therefore, only studies that have purposefully stabilized MTs against depolymerization have detected MTs in spines by EM (Westrum and Gray, 1976; Westrum et al., 1980, 1983; Gray et al., 1982; Mitsuyama et al., 2008). Obviously, fixation freezes any dynamic movement in time. Therefore, if only $\sim 1 \%$ of spines possess MTs at a time when MTs 
are stabilized against depolymerization (this study) it is very unlikely MTs would be detected in electron micrographs, unless several hundred spines were examined in serial sections. Similarly, the lack of detection of MAP2 in spines in time-lapse microscopy (Kaech et al., 2001) could be due to the fact that MAP2 does not label the dynamic ends of MTs (data not shown), but rather the more stable sections of MT polymers that are present in the dendritic shaft. Therefore, dynamic imaging or labeling dendrites with antibodies to MAP2 would not show MTs in spines. This suggestion is consistent with the lack of movement of EGFPMAP2c in the dendrite shaft by time-lapse imaging (Kaech et al., 2001), although a population of MTs is very dynamic in dendrite shafts of mature Purkinje (Stepanova et al., 2003) and hippocam$\mathrm{pal} /$ cortical neurons (this study). Thus, the aforementioned studies are not inconsistent with the present results.

\section{Potential functions of MT invasion of dendritic protrusions}

MTs target spines more frequently and for longer periods of time than stable filopodia. These results suggest that MT invasion of spines may function in maintaining spine structure. However, it does not preclude the possibility that MT invasion of filopodia may be important for maturation of filopodia into spines (Hayashi and Majewska, 2005; Alvarez and Sabatini, 2007; Bourne and Harris, 2007; Harms and Dunaevsky, 2007). This hypothesis is consistent with the results from Zheng and colleagues ( $\mathrm{Gu}$ et al., 2008) in which they show that inhibiting MT dynamics throughout the neuron inhibits BDNF-induced increases in spine numbers and knock-down of the +TIP protein EB3 with shRNA also decreases spine numbers.

Because MTs are the main route of transport of materials from the cell body to peripheral neuronal processes their transient invasion of dendritic spines could be an important pathway for delivery of material to nascent and established dendritic spines. Although all of the MT invasions we documented were transient, they were more than long enough (avg. time in spines $\sim 2.5 \mathrm{~min}$.) for kinesin to transport material into spines and dynein/kinesin to transport material out of spines before MT depolymerization (Vale and Fletterick, 1997). Furthermore, we found MTs enter spines from both proximal and distal regions of the dendrite. Thus, it is likely that anterogradely as well as retrogradely transported material can enter spines via MTs. This result is intriguing because MT-based transport could provide a direct route for communication between distal and proximal spines, as well as between the cell body and specific spines. Presumably, any material that is transported on MTs (Hirokawa and Takemura, 2005) could be directed to specific spines via MTs that polymerize from the dendrite shaft directly into the distal extent of the spine. Indeed, it is tempting to speculate that the dynamic nature of postsynaptic protein redistribution (Gray et al., 2006; Tsuriel et al., 2006) may depend at least in part on MT dynamics.

The function that tSHPs play in synaptic activity is unclear. Protrusions from the spine head, in the form of spinules (Westrum and Blackstad, 1962; Toni et al., 1999; Spacek and Harris, 2004), spine head protrusions (SHPs) (Richards et al., 2005), and lazypodia (Zha et al., 2005) have been documented previously. Oftentimes, these structures are associated with pronounced activity, in the form of LTP induction (Toni et al., 1999), focal application of glutamate (Richards et al., 2005) or bath application of a $\mathrm{GABA}_{\mathrm{A}}$ inhibitor to induce epileptiform activity (Zha et al., 2005). However, spine head protrusions also form after application of tetrodotoxin (TTX), which reduces neuronal activity (Richards et al., 2005). Because tSHPs that we document here are so ephemeral (average lifetime of $40 \mathrm{~s}$ ) and rare (only 4 of 69 spines showed tSHPs) they may differ from spinules, SHPs and lazypodia. Furthermore, we did not detect any transendocytosis of MTs/tubulin, as has been shown for spinules (Spacek and Harris, 2004), suggesting spinules and tSHPs are distinct structures. Further studies will be required to discern the function of these structures and there dependence on MT and actin dynamics.

We document here for the first time that neuronal activity affects the ability of MTs to polymerize into spines. We found that inducing global activity in hippocampal cultures by an established technique (Wu et al., 2001; Li et al., 2004) increased the number of dendritic protrusions that contained a MT by $>400 \%$ ( $0.7 \%$ before $\mathrm{KCl}$ treatment and $3.1 \%$ after $\mathrm{KCl}$ treatment). If we blocked action potential-derived activity with TTX we abolished the ability of $\mathrm{KCl}$ to induce increased invasion of MTs into dendritic protrusions. Furthermore, we discovered MTs that polymerized into these protrusions remained in the protrusions $>250 \%$ longer (3:03 min before $\mathrm{KCl}$ and 7:48 min after). These data show that global activity increases both the number of protrusions targeted by MTs and the time MTs remain in protrusions. Interestingly, we found that most spines that had MT invasion before global activation did not have any MT invasion after activation. Furthermore, most spines that had MT invasions after activation did not have any MT invasions before activation (at least in the $30 \mathrm{~min}$ before $\mathrm{KCl}$ treatment). These data, along with the data showing TTX decreases MT invasions of dendritic protrusions, suggest that MT invasion may coincide with a specific amount of synaptic activation. Further studies are needed to determine whether focal activity is sufficient to cause MT polymerization into individual activated spines and what mechanisms are necessary for MT invasion of spines.

\section{References}

Alvarez VA, Sabatini BL (2007) Anatomical and physiological plasticity of dendritic spines. Annu Rev Neurosci 30:79-97.

Baas PW, Deitch JS, Black MM, Banker GA (1988) Polarity orientation of microtubules in hippocampal neurons: uniformity in the axon and nonuniformity in the dendrite. Proc Natl Acad Sci U S A 85:8335-8339.

Bourne J, Harris KM (2007) Do thin spines learn to be mushroom spines that remember? Curr Opin Neurobiol 17:381-386.

Calabrese B, Wilson MS, Halpain S (2006) Development and regulation of dendritic spine synapses. Physiology (Bethesda) 21:38-47.

Chapleau CA, Carlo ME, Larimore JL, Pozzo-Miller L (2008) The actions of BDNF on dendritic spine density and morphology in organotypic slice cultures depend on the presence of serum in culture media. J Neurosci Methods 169:182-190.

Chicurel ME, Harris KM (1992) Three-dimensional analysis of the structure and composition of CA3 branched dendritic spines and their synaptic relationships with mossy fiber boutons in the rat hippocampus. J Comp Neurol 325:169-182.

Dent EW, Kalil K (2001) Axon branching requires interactions between dynamic microtubules and actin filaments. J Neurosci 21:9757-9769.

Dent EW, Callaway JL, Szebenyi G, Baas PW, Kalil K (1999) Reorganization and movement of microtubules in axonal growth cones and developing interstitial branches. J Neurosci 19:8894-8908.

Dent EW, Tang F, Kalil K (2003) Axon guidance by growth cones and branches: common cytoskeletal and signaling mechanisms. Neuroscientist 9:343-353.

Dent EW, Barnes AM, Tang F, Kalil K (2004) Netrin-1 and semaphorin 3A promote or inhibit cortical axon branching, respectively, by reorganization of the cytoskeleton. J Neurosci 24:3002-3012.

Dent EW, Kwiatkowski AV, Mebane LM, Philippar U, Barzik M, Rubinson DA, Gupton S, Van Veen JE, Furman C, Zhang J, Alberts AS, Mori S, Gertler FB (2007) Filopodia are required for cortical neurite initiation. Nat Cell Biol 9:1347-1359.

Ethell IM, Pasquale EB (2005) Molecular mechanisms of dendritic spine development and remodeling. Prog Neurobiol 75:161-205.

Fiala JC, Kirov SA, Feinberg MD, Petrak LJ, George P, Goddard CA, Harris KM (2003) Timing of neuronal and glial ultrastructure disruption dur- 
ing brain slice preparation and recovery in vitro. J Comp Neurol 465:90-103.

Goldberg DJ, Burmeister DW (1992) Microtubule-based filopodium-like protrusions form after axotomy. J Neurosci 12:4800-4807.

Gray EG, Westrum LE, Burgoyne RD, Barron J (1982) Synaptic organisation and neuron microtubule distribution. Cell Tissue Res 226:579-588.

Gray NW, Weimer RM, Bureau I, Svoboda K (2006) Rapid redistribution of synaptic PSD-95 in the neocortex in vivo. PLoS Biol 4:e370.

Gu J, Firestein BL, Zheng JQ (2008) Microtubules in dendritic spine development. J Neurosci 28:12120-12124.

Harms KJ, Dunaevsky A (2007) Dendritic spine plasticity: looking beyond development. Brain Res 1184:65-71.

Hayashi Y, Majewska AK (2005) Dendritic spine geometry: functional implication and regulation. Neuron 46:529-532.

Hirokawa N, Takemura R (2005) Molecular motors and mechanisms of directional transport in neurons. Nat Rev Neurosci 6:201-214.

Kaech S, Parmar H, Roelandse M, Bornmann C, Matus A (2001) Cytoskeletal microdifferentiation: a mechanism for organizing morphological plasticity in dendrites. Proc Natl Acad Sci U S A 98:7086-7092.

Kalil K, Szebenyi G, Dent EW (2000) Common mechanisms underlying growth cone guidance and axon branching. J Neurobiol 44:145-158.

Lebrand C, Dent EW, Strasser GA, Lanier LM, Krause M, Svitkina TM, Borisy GG, Gertler FB (2004) Critical role of Ena/VASP proteins for filopodia formation in neurons and in function downstream of netrin-1. Neuron 42:37-49.

Li Z, Okamoto K, Hayashi Y, Sheng M (2004) The importance of dendritic mitochondria in the morphogenesis and plasticity of spines and synapses. Cell 119:873-887.

Mitchison T, Kirschner M (1984) Dynamic instability of microtubule growth. Nature 312:237-242.

Mitsuyama F, Niimi G, Kato K, Hirosawa K, Mikoshiba K, Okuya M, Karagiozov K, Kato Y, Kanno T, Sanoe H, Koide T (2008) Redistribution of microtubules in dendrites of hippocampal CAl neurons after tetanic stimulation during long-term potentiation. Ital J Anat Embryol 113:17-27.

Osumi N, Inoue T (2001) Gene transfer into cultured mammalian embryos by electroporation. Methods 24:35-42.

Richards DA, Mateos JM, Hugel S, de Paola V, Caroni P, Gähwiler BH, McKinney RA (2005) Glutamate induces the rapid formation of spine head protrusions in hippocampal slice cultures. Proc Natl Acad Sci U S A 102:6166-6171.

Schaefer AW, Kabir N, Forscher P (2002) Filopodia and actin arcs guide the assembly and transport of two populations of microtubules with unique dynamic parameters in neuronal growth cones. J Cell Biol 158:139-152.

Schneckenburger H (2005) Total internal reflection fluorescence microscopy: technical innovations and novel applications. Curr Opin Biotechnol 16:13-18.

Spacek J, Harris KM (2004) Trans-endocytosis via spinules in adult rat hippocampus. J Neurosci 24:4233-4241.

Stepanova T, Slemmer J, Hoogenraad CC, Lansbergen G, Dortland B, De Zeeuw CI, Grosveld F, van Cappellen G, Akhmanova A, Galjart N (2003) Visualization of microtubule growth in cultured neurons via the use of EB3-GFP (end-binding protein 3-green fluorescent protein). J Neurosci 23:2655-2664.

Tada T, Sheng M (2006) Molecular mechanisms of dendritic spine morphogenesis. Curr Opin Neurobiol 16:95-101.

Toni N, Buchs PA, Nikonenko I, Bron CR, Muller D (1999) LTP promotes formation of multiple spine synapses between a single axon terminal and a dendrite. Nature 402:421-425.

Tsuriel S, Geva R, Zamorano P, Dresbach T, Boeckers T, Gundelfinger ED, Garner CC, Ziv NE (2006) Local sharing as a predominant determinant of synaptic matrix molecular dynamics. PLoS Biol 4:e271.

Vale RD, Fletterick RJ (1997) The design plan of kinesin motors. Annu Rev Cell Dev Biol 13:745-777.

van Rossum D, Hanisch UK (1999) Cytoskeletal dynamics in dendritic spines: direct modulation by glutamate receptors? Trends Neurosci 22:290-295.

Westrum LE, Blackstad TW (1962) An electron microscopic study of the stratum radiatum of the rat hippocampus (regio superior, CA 1) with particular emphasis on synaptology. J Comp Neurol 119:281-309.

Westrum LE, Gray EG (1976) Microtubules and membrane specializations. Brain Res 105:547-550.

Westrum LE, Jones DH, Gray EG, Barron J (1980) Microtubules, dendritic spines and spine appratuses. Cell Tissue Res 208:171-181.

Westrum LE, Gray EG, Burgoyne RD, Barron J (1983) Synaptic development and microtubule organization. Cell Tissue Res 231:93-102.

Wu GY, Deisseroth K, Tsien RW (2001) Spaced stimuli stabilize MAPK pathway activation and its effects on dendritic morphology. Nat Neurosci 4:151-158.

Zha XM, Green SH, Dailey ME (2005) Regulation of hippocampal synapse remodeling by epileptiform activity. Mol Cell Neurosci 29:494-506.

Zhou FQ, Waterman-Storer CM, Cohan CS (2002) Focal loss of actin bundles causes microtubule redistribution and growth cone turning. J Cell Biol 157:839-849. 